Pesq. Vet. Bras. 30(4):335-339, abril 2010

\title{
Avaliação ultrassonográfica do meato acústico em cães ${ }^{1}$
}

\author{
Polyanne Cramer Schmidlin ${ }^{2}$, Tilde Rodrigues Froes ${ }^{3^{*}}$, Daniela Aparecida \\ Ayres Garcia ${ }^{4}$, Helber Parchen ${ }^{4}$ e Fabiano Montiani-Ferreira ${ }^{3}$
}

\begin{abstract}
Schmidlin P.C., Froes T.R., Garcia D.A.A., Parchen H. \& Montiani-Ferreira F. 2010. [Ultrasonographic evaluation of the external ear in dogs.] Avaliação ultrassonográfica do meato acústico em cães. Pesquisa Veterinária Brasileira 30(4):335339. Departamento de Medicina Veterinária, Universidade Federal do Paraná, Rua dos Funcionários 1540, Curitiba, PR 80035-050, Brazil. E-mail: tilde@ufpr.br

The purpose of this study was to determine the viability of ultrasonographic evaluation of the external ear in dogs. The thickness and architecture of the ear wall and tympanic bulla of 10 Labrador Retrievers without ear disease was evaluated. The examination was performed with an ultrasound system and a $14 \mathrm{MHz}$ bidimentional linear-array transducer and general anesthesia, before and after the infusion of saline solution into the ear canal. The maxillary artery was identified with color Doppler and helped to establish the location of the tympanic bulla in all dogs. The proximal wall was less well-defined when compared with the distal wall of the ear canal. Dogs with increased ceruminal content within the ear canal showed a hypoechoic epithelium. The tympanic membrane was not identified. It was concluded that ultrasound evaluation is feasible and needs to be explored as clinical diagnostic tool for the diagnosis of ear diseases in dogs.
\end{abstract}

INDEX TERMS: Ultrasonography, external ear canal, tympanic bulla, dogs.

RESUMO.- O propósito deste trabalho foi determinar a viabilidade da ultrassonografia para avaliar o canal auditivo externo de cães, aferindo a espessura e arquitetura da parede do conduto auditivo e bula timpânica. Foram avaliados 10 cães da raça Retriever do Labrador não portadores de otite externa. O exame foi realizado com o aparelho de ultrassonografia bidimensional e transdutor linear de $14 \mathrm{MHz}$, com todos os animais sob efeito de anestesia geral. O exame foi efetivado antes e depois do conduto ser preenchido com solução salina, promovendo uma janela acústica anecóica. A artéria maxilar foi identificada com o Doppler colorido o que facilitou a localização da bula timpânica em todos os cães. A definição da imagem da parede proximal ao transdutor foi pior quando comparada à parede distal $\mathrm{fem}$ todos os animais, isto

\footnotetext{
${ }^{1}$ Recebido em 19 de janeiro de 2009.

Aceito para publicação em 1 de dezembro de 2009.

${ }^{2}$ Mestranda do Programa de Pós-Graduação em Ciências Veterinárias, Universidade Federal do Paraná (UFPR), Rua dos Funcionários 1540, Curitiba, PR 80035-050, Brasil.

${ }^{3}$ Departamento de Medicina Veterinária, UFPR, Curitiba, PR. *Autor para correspondência: tilde@ufpr.br

4. Médicos Veterinários em Curso de Especialização, Hospital Veterinário, UFPR, Curitiba, PR.
}

devido à proximidade da parede com a face de leitura do transdutor. Nos cães que apresentavam uma maior quantidade de cerúmen a superfície do epitélio estava com uma camada hiperecóica mais evidente. A membrana timpânica não foi identificada em nenhum animal. Conclui-se que ultrassonografia é viável e deve ser mais pesquisada na contribuição diagnóstica das doenças óticas em cães.

TERMOS DE INDEXAÇÃO: Ultrassonografia, conduto auditivo, bula timpânica, canino.

\section{INTRODUÇÃO}

A orelha do cão compõe-se de três partes: a orelha externa, a média e a interna (Heine 2004). Juntos, esses componentes permitem que o animal localize o som e a direção de onde ele provém, e oriente a cabeça em relação à gravidade, como também aceleração e rotação (Getty 1981). Existe ampla variação no tamanho relativo e na forma dos componentes da orelha dos cães resultante da excessiva reprodução seletiva e variedade racial desta espécie. A forma e a posição da orelha, o diâmetro do canal auditivo externo, a quantidade de pêlos e de tecido mole no interior do canal varia de uma raça para outra (Harvey et al. 2004). 
O meato que conduz as ondas sonoras até o tímpano é composto pela porção vertical e horizontal. O meato auditivo externo proximal inicia-se na membrana timpânica e o distal termina junto dos componentes cartilaginosos da base da orelha externa (Harvey et al. 2004).

A membrana timpânica separa a orelha externa da média e está locada na câmara dorsal da bula timpânica, região que são encontrados os três ossículos timpânicos (martelo, bigorna e estribo), e a janela da cóclea, estrutura que divide a orelha média da orelha interna. A tuba auditiva (de Eustáquio) se insere da região rostral da bula e se estende até a nasofaringe e é também denominada de conduto auditivo interno (Getty 1981, Cole 2004).

Uma das formas mais diretas na avaliação do conduto auditivo e da membrana timpânica é a otoscopia, a qual permite a avaliação direta do canal auditivo. Essa deve ser realizada com cones veterinários adequados do tamanho auricular de cada paciente (Bruyette 1993). Para uma perfeita avaliação se faz necessária a contenção do paciente com sedação superficial ou profunda, pois o canal auditivo precisa ser manipulado de forma reta, o que se consegue pela manobra de tracionar a orelha, puxando-a, junto com a cartilagem auricular anexa, para cima e para longe do plano sagital, causando desconforto ao paciente (Harvey et al. 2004). Pela otoscopia distinguese o tipo de secreção, que ainda, pode ser coletada para análises laboratoriais. Além da possibilidade de se detectar parasitas, corpos estranhos, ou outras alterações do epitélio e diâmetro do canal, incluindo processos neoplásicos (Bruyette 1993, Scott et al. 1996). Devido à estenose acentuada de alguns canais auditivos, ou excesso de pêlos e secreção dentro do conduto, o exame otoscópio nem sempre pode ser realizado de uma maneira efetiva, principalmente no que tange a análise da integridade da membrana timpânica. Como também, o fato da membrana timpânica se encontrar intacta na realização do exame, não descarta a possibilidade do paciente ter otite média. Sendo assim, outras técnicas imaginológicas se tornam necessárias para uma avaliação mais precisa do conduto auditivo e da bula timpânica.

Diferentes técnicas de imagem podem ser utilizadas na avaliação das doenças do canal auditivo, cada uma delas com contribuições específicas e diferentes limitações, entre estas se encaixam: radiologia simples, radiologia contrastada, tomografia computadorizada e ressonância nuclear magnética (Bischoff \& Kneller 2004).

O exame radiográfico tem como principal objetivo a análise dos casos crônicos, especialmente, para tentarse determinar o grau de otite média (Holt \& Walker 1997). Todavia, projeções específicas como, por exemplo, a rostrocaudal de boca aberta são recomendadas para observação das bulas timpânicas, o que geralmente exige a necessidade de anestesia geral. Sabe-se ainda que devido à complexidade anatômica da bula timpânica resultados falsos negativos são observados em 25 a 30\% dos pacientes (Griffiths 2003). Sem a utilização de técnicas de contraste não é possível avaliar a integridade da mem- brana timpânica, nem visualizar a posição de uma neoplasia luminal obstrutiva (Harvey et al. 2004). Embora para o diagnóstico de otite média, a radiografia tenha se mostrado menos sensível que a cirurgia ou a tomografia computadorizada, sua importância não deve ser subestimada (Doust et al. 2007).

Alguns pesquisadores citaram a tomografia computadorizada (TC) como uma modalidade alternativa de meIhor avaliação do conduto auditivo. Contudo, a técnica é de 20 a 30 minutos mais demorada quando comparada ao exame radiográfico não possui um aumento significativo na resolução da imagem. A verdadeira contribuição dessa técnica ainda é discutivel. Em estudo que compara a radiografia com a TC para o diagnóstico de otite média, os autores concluíram que a TC forneceu muito pouca informação adicional para que se justifiquem os seus custos. Apesar de mais sensível, a mesma é menos específica. Nessa mesma pesquisa, foi possível comprovar que a radiografia e a TC foram incapazes de detectar alterações recentes de otite média, em que ainda não havia alterações ósseas (Rohleder et al. 2006).

A ressonância magnética foi considerada por Dvir et al. (2000) o "padrão ouro" para o diagnóstico de otite média, porém sua utilização ainda é bastante limitada à maioria dos médicos veterinários em rotina clínica. Apesar de a técnica permitir uma perfeita avaliação dos tecidos moles do conduto auditivo, a mesma não oferece resolução superior para lesões ósseas, sendo essa a sua limitação, tornando-a também complementar a TC para tais análises.

A ultrassonografia é um exame que tem inúmeras aplicações nos mais variados sistemas orgânicos, porém sua utilização para avaliação do ouvido externo e médio ainda não está totalmente estabelecida. Assim como os outros métodos imaginológicos, a ultrassonografia não é invasiva. Suas grandes vantagens são inerentes a sua característica física, estando relacionada ao baixo custo, não invasividade e a não utilização de radiação ionizante (Dickie et al. 2003).

Poucos estudos foram realizados com o objetivo de determinar a contribuição dessa modalidade na avaliação do conduto auditivo em cães. Griffiths et al. (2003) descreveram a utilização da ultrassonografia versus a radiografia na detecção de fluido na bula timpânica, nessa investigação comprovou-se que o método ultrassonográfico foi $100 \%$ sensível e específico na detecção de fluido na bula timpânica, sendo melhor que o exame radiográfico que teve $80 \%$ de sensibilidade e $65 \%$ de especificidade. Ou seja, os pesquisadores concluíram que a técnica é passiva de realização e pode adicionar informações no diagnóstico do paciente com otite.

Lee et al. (2006) descreveram os padrões de normalidade sonográfica em Beagles hígidos e Beagles com ruptura da membrana timpânica iatrogênica, esses autores findam que a técnica é simples, não invasiva e possibilita a perfeita avaliação do canal auditivo. Sendo que, a partir desses achados, acredita-se ser plausível a sua uti- 
lização também na avaliação do meato auditivo para diferentes enfermidades, como por exemplo: excesso de pêlo ou cerume, corpos estranhos, alterações proliferativas, pólipos, estenoses e neoplasias.

O objetivo desse estudo foi avaliar os padrões anatômicos de normalidade sonográfica do meato auditivo, em cães hígidos da raça Retriever do Labrador, bem como, analisar a sua executabilidade, dificuldades e possíveis contribuições, comparando então estes dados com outras pesquisas previamente realizadas.

\section{MATERIAL E MÉTODOS}

Foram avaliados 10 cães da raça Retriever do Labrador não portadores de otite externa, com peso de 23-30 kg. O exame foi realizado com o aparelho de ultrassonografia eletrônico, bidimensional de alta resolução (Modelo SONIX OP, marca Ultrasonix (Viking Way, Richmond, BC, Canadá) e transdutor linear de $14 \mathrm{MHz}$ com todos os animais sob efeito de anestesia geral. Foi realizada tricotomia de toda região adjacente à orelha externa, com os cães posicionados em decúbito lateral com o pescoço estendido (Fig.1). O exame ultrassonográfico era realizado antes e depois da aplicação de solução salina 0,9\% dentro do meato auditivo (Fig.2A,B). A solução salina foi aquecida até atingir a temperatura corporal antes de ser infundida no meato auditivo por meio de uma sonda uretral de número seis. $O$ meato vertical era massageado- e em seguida infundia-se mais solução salina até atingir o nível do trago. Para o exame o transdutor foi posicionado lateralmente sobre a parede lateral do conduto auditivo externo promovendo-se a janela para um corte longitudinal do conduto vertical e seguidamente movido ventralmente para avaliar a porção horizontal do conduto. Em seguida girou-se o transdutor $10^{\circ} \mathrm{a}$ $20^{\circ}$ para avaliar o canal e a bula timpânica no mesmo plano de imagem. Para a obtenção de um plano transversal de imagem, o transdutor era posicionado acima do canal vertical, perpendicularmente ao plano sagital do crânio. O exame com Doppler colorido foi utilizado para auxiliar na identificação da artéria maxilar, o que definitivamente facilitou a localização da bula timpânica em todos os animais.

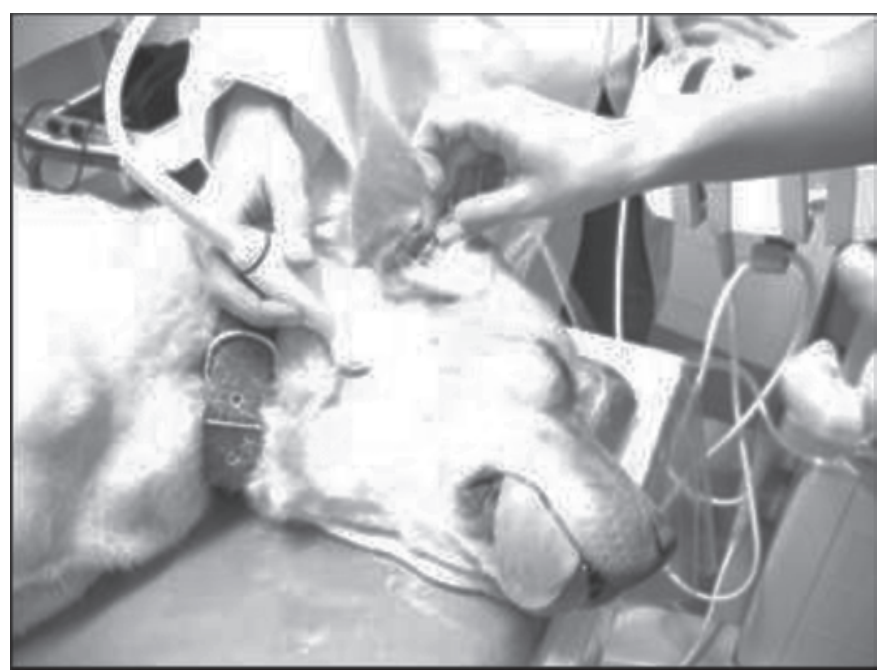

Fig.1. Cão posicionado para o exame. Note o posicionamento do caudo-sagital do transdutor em relação ao trago.

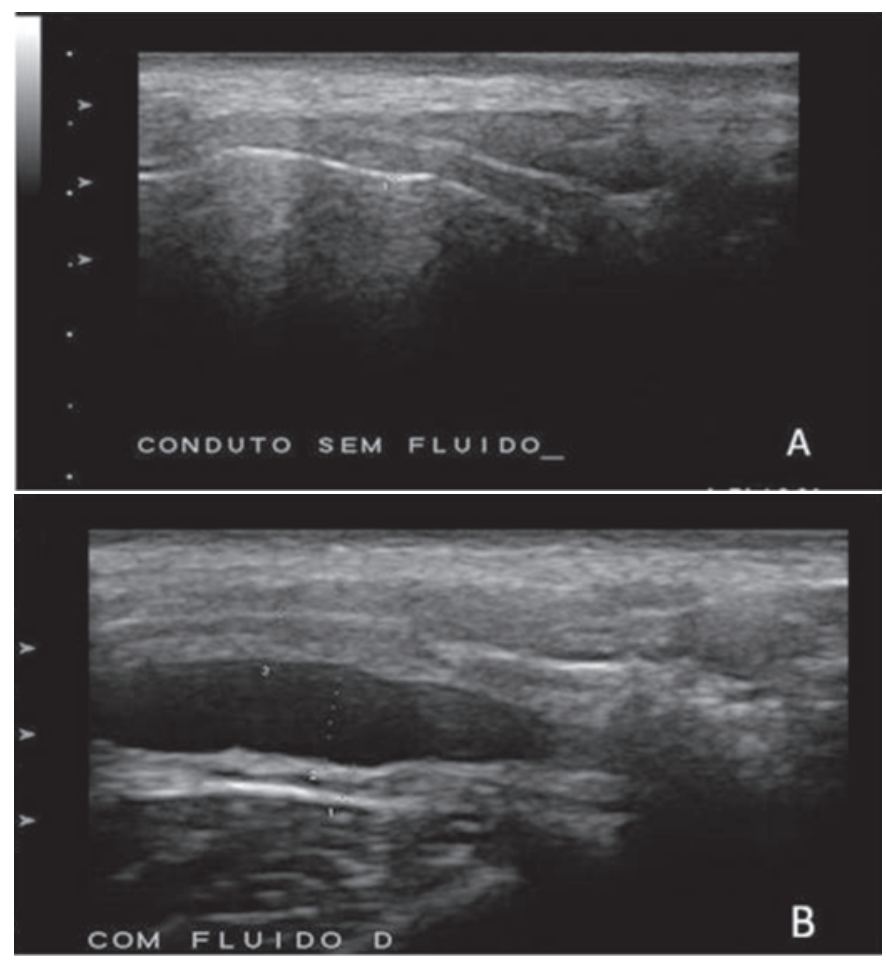

Fig.2. Imagens ultrassonográficas, (A) do corte longitudinal do conduto auditivo anteriormente ao seu preenchimento com fluído aquecido, (B) após o preenchimento com fluído aquecido.

\section{RESULTADOS E DISCUSSÃO}

Apesar do baixo custo o exame ultrassonográfico nos traz informações relevantes da arquitetura da parede e lúmen do conduto auditivo, permitindo também uma perfeita estimativa do conteúdo e canal luminal, podendo ser mais um método diagnóstico imaginológico nos casos de otite crônica, assim como citado por Lee et al. (2006). Suas vantagens previamente citadas devem sempre ser correlacionadas com as demais modalidades de diagnósticas por imagem, basicamente se considerarmos, que todas têm como desvantagem a necessidade de sedação e anestesia geral para perfeita avaliação. (Dvir et al. 2000, Dickie et al. 2003, Griffithis et al. 2003, Rohleder et al. 2006).

Ou seja, essa também é a aparente desvantagem do exame sonográfico, no qual deve ser realizado sob efeito de anestesia geral, pois sem tal efeito foi difícil o preenchimento do meato auditivo pela solução fisiológica em todos os animais deste experimento. Se for considerado que para uma avaliação otoscópica satisfatória o procedimento anestésico também é recomendado, como citado por Scott et al. (1996), a adição das duas técnicas talvez seja a melhor opção. Vale ponderar que uma das formas de tratamento em pacientes portadores de otite crônica seria a lavagem auricular, com irrigação e aspiração de solução fisiológica (Scott et al. 1996), no qual seria então realizada posteriormente ao exame ultrassonográfico, não trazendo desta forma prejuízos ao paciente no 
que tange a realizar a anestesia, e sim somente contribuições.

Vislumbra-se, por que então, não se realizar a combinação das três técnicas, no qual uma contribuiria com a outra para completa análise do conduto auditivo em todos os seus planos, isso sendo realizado em um mesmo tempo anestésico.

Pela análise deste trabalho, constatou-se ainda que o procedimento é rápido, não perdurando mais que $15 \mathrm{mi}-$ nutos, o que tornaria a duração anestésica reduzida, mesmo se considerar a análise prévia ou posterior pela otoscopia.

Em todos os exames verificou-se que o preenchimento do canal por solução fisiológica foi imprescindível para análise ultrassonográfica, assim como sugerido por Lee et al. (2006).

Em seguida ao preenchimento com fluído, houve boa delimitação ultrassonográfica de toda a extensão da parede proximal e distal do conduto auditivo, bem como, a identificação de suas camadas. A camada externa foi visibilizada como uma camada, fina e hiperecóica; a camada interna era a mais espessa e de fácil delimitação apresentando-se hipoecóica. O lúmen apresentava-se anecóico com pontos ecogênicos em suspensão devido ao seu preenchimento pelo fluido, podendo o mesmo ser comprimido dependendo da força da pressão manual do transdutor (Fig.3). A espessura da parede do conduto auditivo variou de $1,47-2,04 \mathrm{~mm}$ para parede distal e de $1,85-2,72 \mathrm{~mm}$ para a parede proximal ao transdutor. Estes dados devem ser considerados unicamente para os cães da raça Retriever do Labrador, já que, provavelmente a espessura da parede, a extensão do canal e o diâmetro do lúmen variam de acordo também com a raça do animal, dados estes correlacionados às variações dos padrões anatômicos previamente indicados por demais pesquisadores (Harvey et al. 2004).

A definição da imagem da parede proximal ao transdutor foi pior do que a parede distal ao transdutor em

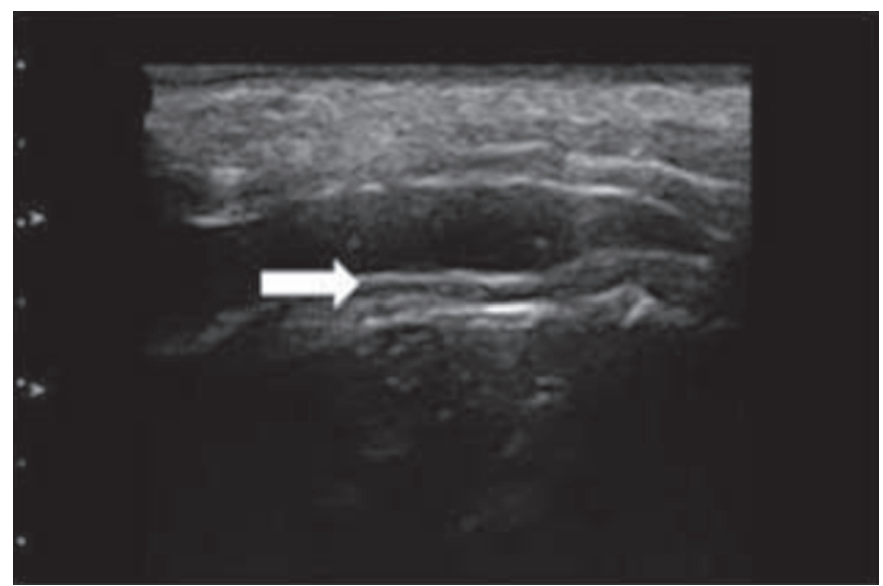

Fig.3. Imagem ultrassonográfica da parede do meato auditivo, hipoecóica com porção face do epitélio hiperecóico (seta) em um cão com excesso de cerúmen.

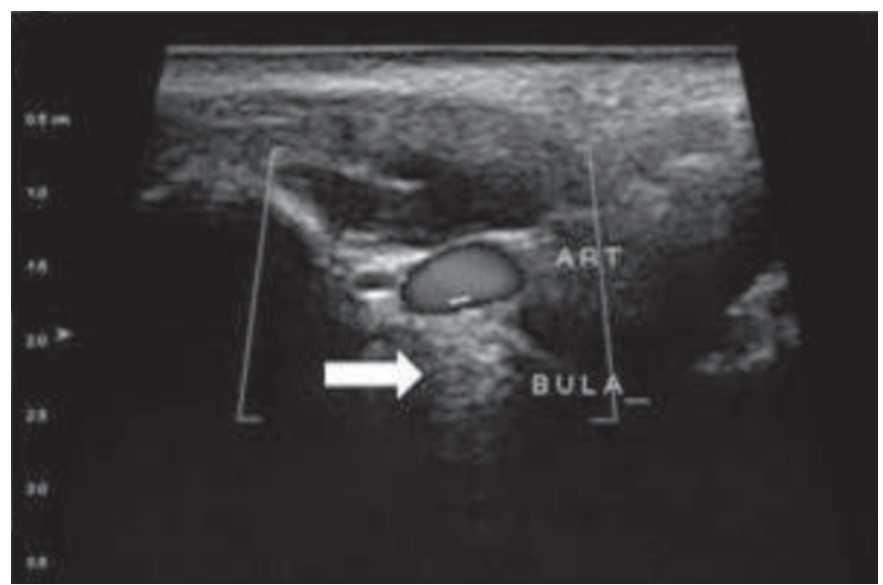

Fig.4. Imagem sonográfica da bula timpânica localizada após a identificação da artéria maxilar pelo Doppler colorido (seta).

todos os animais mesmo se considerar a alta freqüência do transdutor utilizado, dificuldade esta não reportada por Dickie et al. (2003) e por Lee et al. (2006).

A maior dificuldade da visibilização da parede do conduto mais próximo ao transdutor se deve a discreta quantidade de ar intraluminal retida, mesmo após a administração do conteúdo líquido, produzindo um pequeno artefato de reverberação, todavia, não limitando o exame desta porção. Nos cães que apresentavam uma maior quantidade de cerúmen a superfície da parede era mais evidente e hiperecóica Mais uma vez, ressalta-se que cuidados devem ser tomados na avaliação do lúmen, já que pode haver deformidade do seu diâmetro devido à pressão externa do transdutor.

Em consonância com Lee et al. (2006), verificou-se que a bula timpânica foi visibilizada em todos os animais, sendo identificada por meio da proximidade com a artéria maxilar. A identificação da artéria maxilar foi facilitada pela técnica do Doppler colorido (Lee et al. 2006), a mesma foi utilizada como ponto de referência anatômica para visibilização das bulas (Fig.4). Em todos os animais identificaram-se as bulas timpânicas, sendo que o diâmetro aproximado, nos cães variou de 4,5-6,7mm de diâmetro. Outros autores utilizaram Beagles saudáveis, porem não reportaram suas medidas (Lee et al. 2006). A membrana timpânica não foi visibilizada em nenhum animal, determinando-se que a mesma só pode ser identificada provavelmente quando rompida ou por meio da movimentação de líquido após a infusão de solução dentro da bula com a membrana intacta, fato este citado por Lee et al. (2006).

Após três dias, a otoscopia dos animais revelou ausência de umidade nos condutos auditivos e nenhum cão desenvolveu otite externa após o exame ultrassonográfi$\mathrm{co}$, demonstrando que nas condições em que foi realizada esta pesquisa, tal modalidade diagnóstica não oferece riscos de desenvolver doenças iatrogênicas.

Assim com mencionado por Griffiths et al. (2003), Dickie et al. (2003) e Lee et al. (2006) comprovou-se neste estudo que a avaliação do conduto auditivo de cães é possível pela técnica ultrassonográfica, contudo, é impres- 
cindível o preenchimento do conduto auditivo com solução salina, para de tal forma haver a produção de uma janela acústica.

\section{CONCLUSÃO}

Por este estudo dentro da metodologia proposta, pode-se concluir que a ultrassonografia é viável para avaliação do conduto auditivo abrindo-se novas perspectivas de estudos em animais portadores de doenças óticas.

\section{REFERÊNCIAS}

Bischoff M.G. \& Kneller S.K. 2004. Diagnostic imaging of the canine and feline ear. Vet. Clin. North Am., Small. Anim. Pract. 34(2):437-459.

Bruyette D.S. \& Lorenz M.D. 1993. Otitis externa and otitis media: Diagnostic and medical aspect. Semin Vet Med. Surg Small Anim. 8(1):3-9.

Cole K.L. 2004. Otoscoic evaluation of the ear canal. Vet. Clin. North Am., Small Anim. Pract. 34(2):397-410.

Dickie A.M., Doust R., Cromarty L., Johnson V.S., Sullivan M. \& Boyd J.S. 2003. Ultrasound imaging of the canine bulla. Res. Vet. Sci. 75(2):121-126.

Doust R., King G., Hammond T., Weirauch S., Mellor D. \& Sullivan M. 2007. Assessment of middle ear disease in the dog: A comparison of diagnostic imaging modalities. J. Small Anim. Pract. 48(4):188-192.
Dvir E., Kirberger R.M. \& Terblanche A.G. 2000. Magnetic resonance imaging of otitis media in a dog. Vet. Radiol. Ultrasound. 41(1):4649.

Getty R. 1981. Sisson/Grossman, Anatomia dos Animais Domésticos. 5 ${ }^{a}$ ed. W.B. Saunders, Philadelphia, p.1660-1669.

Griffths L.G., Sullivan M., O’Neill T. \& Reid S.W.J. 2003. Ultrasonography versus radiography for detection of fluid in the canine tympanic bulla. Vet. Radiol. Ultrasound 44(2):210-213.

Harvey R.G., Harai J. \& Deluxe A. 2004 Procedimentos para diagnóstico, p.70-72. In: Ibid. (Eds), Doenças do Ouvido em Cães e Gatos. Revinter, Castelo Branco, Portugal.

Heine A.P. 2004 Anatomy of the ear. Vet. Clin. North Am., Small Anim. Pract. 34(2):379-395.

Holt D.E. \& Walker L. 1997. Radiographic appearance of the middle ear after ventral bulla osteotomy in five dogs with otitis media. Vet. Radiol. Ultrasound 38(3):182-184.

Lee J., Eom K., Seong Y., Lee H., Park J., Lee J., Jang K., Lee K., Oh T., Lee S., Yoon J., Lee H., Choi H., Lee Y. \& Chang D. 2006. Ultrasonographic evaluation of the external ear canal and tympanic membrane in dogs. Vet. Radiol. Ultrasound 44(2):210-213.

Rohleder J.J., Jones J.C., Duncan R.B., Larson M.M., Waldron D.L. \& Trombkee T. 2006. Comparative performance of radiography and computed tomography in the diagnosis of middle ear disease in 31 dogs. Vet. Radiol.Ultrasound 47(2):45-52.

Scott D.W., Miller W.H. \& Griffin C.E. 1996. Muller \& Kirk's Small Animal Dermatology. $5^{\text {th }}$ ed. W.B. Saunders, Philadelphia, p.907-923. 